\title{
Chemoembolization Alone or Associated With Bevacizumab for Therapy of Colorectal Cancer Metastases: Preliminary Results of a Randomized Study
}

\author{
GIAMMARIA FIORENTINI ${ }^{1}$, DONATELLA SARTI ${ }^{1}$, MICHELE NARDELLA $^{2}$, RICCARDO INCHINGOLO $^{2}$, \\ MASSIMILIANO NESTOLA ${ }^{2}$, ALBERTO REBONATO ${ }^{3}$ and STEFANO GUADAGNI ${ }^{4}$ \\ ${ }^{1}$ Department of Oncology-Hematology, Azienda Ospedaliera Ospedali Riuniti Marche Nord, Pesaro, Italy; \\ ${ }^{2}$ Diagnostic and Interventional Radiology Department, Ospedale Madonna delle Grazie, Matera, Italy; \\ ${ }^{3}$ Diagnostics for Images Unit and Interventional Radiology, \\ Azienda Ospedaliera Ospedali Riuniti Marche Nord, Pesaro, Italy; \\ ${ }^{4}$ Department of Applied Clinical Sciences and Biotechnology, \\ Section of General Surgery, University of L'Aquila, L'Aquila, Italy
}

\begin{abstract}
Aim: to assess efficacy and safety of chemoembolization alone (TACE) and followed by bevacizumab (TACE-B) in patients with colorectal liver metastases (CRC-LM) (NCT03732235). Patients and Methods: The study included 30 consecutive patients with CRC-LM. They were informed about the types of treatment available: TACE with irinotecan loaded into polythylene glycol embolics alone or followed by bevacizumab therapy. Each patient underwent self-randomization and 17 chose TACE, whereas 13 chose TACE-B. Results: Tumor response at 3 months was complete response in one (6\%) and four (31\%) patients, and partial response in two (13\%) and six (46\%) patients, after TACE and TACE-B, respectively. No complications were observed during TACE. Most TACErelated adverse events were correlated with post-embolic syndrome. Conclusion: The preliminary results of the study showed that the TACE-B is feasible and tolerable. This study will be continued in order accrue a larger number of patients and longer follow-up.
\end{abstract}

This article is freely accessible online.

Correspondence to: Dr. Giammaria Fiorentini, Department of Oncology-Hematology, Azienda Ospedaliera Ospedali Riuniti Marche Nord, San Salvatore Hospital, Via Lombroso 1, 61122 Pesaro, Italy. Tel: +39 0721364005, Fax: +39 0721364094, e-mail: g.fiorentini@alice.it

Key Words: Liver metastases, colorectal carcinoma, chemoembolization, TACE, anti-angiogenesis, bevacizumab, irinotecan, tumor response.
Liver metastases are present in more than $50 \%$ of patients affected by colorectal cancer (CRC) $(1,2)$. Surgical resection of liver metastases from colorectal cancer (CRC-LM) is feasible in only $15-20 \%$ of cases (3). Median survival at 5 years for those with complete resection (R0) is $25-35 \%$, and recurrence in the liver is frequent (1-3). Despite the introduction of new chemotherapeutic agents and the development of locoregional therapies (embolization, percutaneous ablation, hepatic arterial-directed infusion chemotherapy, internal radiation), there is still a lack of standardized evidence-based protocols for optimal CRC-LM management (3). Transarterial chemoembolization (TACE) with irinotecan-loaded polyethylene glycol embolics has produced interesting results in terms of objective response in CRC-LM treatment (4).

There is wide evidence that CRC is angiogenesisdependent and that angiogenesis is elevated in CRC compared to non-neoplastic tissue (5-7). Vascular endothelial growth factor (VEGF) is the most important pro-angiogenic factor and is expressed at higher levels in CRC than in nonneoplastic tissues (8-10). The first anti-angiogenic therapy was introduced in 2004 with a humanized murine monoclonal antibody against VEGF called bevacizumab (10, 11). Bevacizumab is approved for the treatment of CRC-LM in combination with fluoropyrimidine-based chemotherapy $(12,13)$. It is used in normal clinical practice as first-, second- and third-line chemotherapy for CRC-LM and is often associated with chemoembolization.

Expression of VEGF is up-regulated by hypoxia-inducible factor 1 and 2 (HIF1 and HIF2). Chemoembolization induces a hypoxic microenvironment that increases the expression of HIF1 and HIF2, resulting in high VEGF expression and neoangiogenesis. This may also induce tumor relapse. For this 
reason, there is a strong rationale for the association of a biological agent, such as bevacizumab, with hepatic chemoembolization treatment (14).

The aim of this study was to collect data on the treatment of patients with CRC-LM that was refractory to systemic therapies and was treated with TACE alone or in association with bevacizumab, in order to assess tolerability, quality of life and objective responses.

\section{Patients and Methods}

Patients. This was a prospective observational study that was approved by the local Institutional Review Board (ClinicalTrials.gov Identifier: NCT03732235). The study included 30 consecutive patients with CRC-LM. They were informed about the types of treatment available: TACE with irinotecan loaded into polythylene glycol embolics alone or TACE followed by bevacizumab therapy (TACE-B). Each patient underwent self randomization as reported in other studies (15): They were informed about the two types of therapies available and were asked to choose the one that they preferred; 17 chose TACE, whereas 13 chose TACE-B. Patients were included in the study if they met the following inclusion criteria: Age $>18$ years, diagnosis of CRC-LM, unresectable disease, no response to standard chemotherapeutic lines, Eastern Cooperative Oncology Group performance status of 0-1, tumor size evaluable according to RECIST version 1.1 (16), liver involvement from $25 \%$ to $40 \%$, life expectancy of at least 3 months, normal ranges of routine blood biochemistry assays.

Exclusion criteria were the following: Contraindication to angiographic catheterization, extensive extra-hepatic disease, pregnancy or breast feeding, other severe clinical contraindications.

TACE and TACE-B procedures. Tumor arterial perfusion was assessed by diagnostic angiography before TACE. TACE was performed using $2 \mathrm{ml}$ of LifePearl ${ }^{\circledR}$ with 100 micron diameter (Terumo Europe NV, Leuven, Belgium) that were loaded with irinotecan $(100 \mathrm{mg})$ as previously described $(4,13)$. Infusion was performed at fixed speed of $1 \mathrm{ml} /$ minute for a median time of 12 minutes. A second TACE was performed after 30 days.

For the TACE-B group, bevacizumab therapy was started 15 days after the first TACE at a concentration of $5 \mathrm{mg} / \mathrm{kg}$, and was repeated every 2 weeks for a total of eight cycles.

Assessment of objectives. The baseline data collected for each patient included: medical history; weight; height; blood pressure; performance status; routine complete blood chemistry; tumor parameters [carbohydrate antigen 19-9 (CA 19-9), carcinoembryonic antigen (CEA) and VEGF]; tumor assessment by computed tomographic scan of the thorax, abdomen and pelvis; fluorodeoxyglucose positronemission tomography/computed tomography with scan or liver ultrasound contrast agent (Sonovue). RECIST criteria version 1.1 (13) was used to monitor tumor response (at 3 , months) from abdominal and pelvic computed tomographic imaging. The CEA level was monitored at 1, 3, 6 months after TACE.

National Cancer Institute Common Terminology Criteria for Adverse Events (NCI-CTCAE), version 3.0 was used to classify adverse events (16).

Statistical analysis. The data analysis of the whole sample was performed using the median for continuous data; proportions are expressed as percentages. Significance of continuous variables was assessed with chi-square and Student's t-test $(p<0.05)$. Overall survival (OS) was analyzed with the Kaplan-Meier method, and significance was computed with the log rank test, significance was established as $p<0.05$.

\section{Results}

Patients. The study included 30 patients affected by unresectable CRC-LM that were non-responsive to systemic therapy. Seventeen were treated with TACE alone and 13 with TACE-B. Fifteen $(50 \%)$ patients were males and 15 $(50 \%)$ females. The median patient age was 72.5 (range $=50$ 87) years (Table I).

Tumor response. Tumor responses at 3 months after TACE and TACE-B were complete response in one $(6 \%)$ and four (31\%), partial response in two (13\%) and six (46\%), stable disease in eight $(50 \%)$ and two $(15 \%)$, and progressive disease in five $(31 \%)$ and one $(8 \%)$, respectively. The corresponding disease control rates were $69 \%$ and $92 \%$ for TACE and TACE-B, respectively; the difference was statistically significant $(p<0.01)$.

Median follow-up was 6 (range $=1.3-6)$ months. The CEA level consistently decreased from baseline to 1,3 and 6 months (Table I) in all patients.

Survival. Median overall survival (OS) was 12 (range $=7-22$ months) (Figure 1), time to progression was 4.5 (range 3-9) months and median progression-free survival was 6 (range $=3$ 18) months for the TACE-B group. Median OS was 5.8 (range $=1.5-7.7$ ) months (Figure 1), median time to progression was 2.9 (range=1.5-6.4) months and median progression-free survival was 4 (range $=1.5-7.7$ ) months in the TACE group. OS and progression-free survival were statistically greater $(p<0.01)$ in the TACE-B than in the TACE group.

Tolerability. No complications were observed during TACE. Most TACE-related adverse events were correlated with post-embolic syndrome and were of mild or moderate intensity. Pain (grade 2) was observed in 14 (47\%) patients, and was resolved in 2-5 days. Transaminase rise was observed in $10(33 \%)$ patients and was of grade 2-3 intensity. No thromboembolic effects were observed.

Bevacizumab-related adverse events were increased blood pressure (grade 2) in two (16\%), fever (grade 2) in two $(16 \%)$ and skin rash (grade 2$)$ in four $(31 \%)$ patients.

\section{Discussion}

TACE is an occlusive procedure that closes medium-caliber vessels feeding the tumor. Tumors need neovascularization in order to obtain oxygen and nutrients for their growth and development. Bevacizumab creates an insufficient blood 
Table I. Characteristics of study population.

\begin{tabular}{lc}
\hline Characteristic & Value \\
\hline Gender, n (\%) & \\
Male & $15(50 \%)$ \\
Female & $15(50 \%)$ \\
Age, years & \\
Median (range) & $72.5(50-87)$ \\
Tumor size, mm & \\
Median (range) & $50(5-110)$ \\
Tumor nodules, n (\%) & \\
$1-2$ & $7(23 \%)$ \\
$3-5$ & $14(47 \%)$ \\
$>5$ & $9(30 \%)$ \\
CEA, U/ml & \\
Baseline, median (range) & $400(110-932)$ \\
At 1 Month, median (range) & $250(70-800)$ \\
At 3 Months, median (range) & $212(40-763)$ \\
At 6 Months, median (range) & $135(45-245)$ \\
Chemotherapy lines, n (\%) & \\
1 & $4(13 \%)$ \\
2 & $11(37 \%)$ \\
3 & $8(27 \%)$ \\
$>3$ & $7(23 \%)$ \\
\hline
\end{tabular}

afflux to the tumor by the biomolecular mechanism of antiangiogenesis. The association of TACE with bevacizumab creates a double antiangiogenic attack.

TACE can be associated with several anti-angiogenic factors (bevacizumab, aflibercept and regorafenib) in targeted therapy (17). This association can increase tumor responses and duration, and improve quality of life.

Very few studies have focused on the association of TACE with bevacizumab, and they were performed in HCC (18-20).

Bevacizumab has been used with proven results in association with irinotecan, as bolus followed by infusional 5fluorouracil and leucovorin (FOLFIRI) and with irinotecan, bolus fluorouracil and leucovorin, resulting in increased partial response and objective response rates, and reduction of mortality risk in patients with CRC-LM $(19,20)$.

In the present study, we collected data on 30 patients affected by CRC-LM whose disease was refractory to previous chemotherapy and who were treated with TACE alone or followed by intravenous bevacizumab. Median OS was 12 months (range $=7-18$ months) and 5.7 (range=1.5-7.7) months in the TACE and TACE-B groups, respectively. The median OS of the TACE-B group [12 (range=7-22) vs. 5.8 (range $=1.5-7.7)$ months for TACE alone] was comparable to that previously reported for patients with CRC-LM treated with TACE alone [14 (range=1.3-25) months] (21). Many patients included in this study received more than two lines of chemotherapy, this may have influenced OS and reduced their life expectancy.

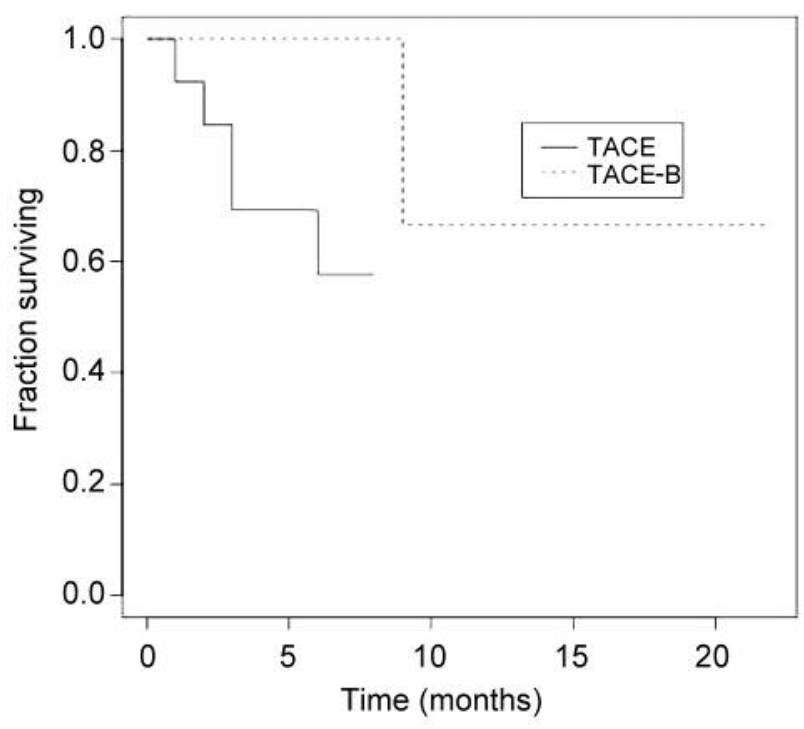

Figure 1. Kaplan-Meier curve of survival according to therapy with transarterial chemoembolization with irinotecan (TACE) alone and combined with bevacizumab (TACE-B).

The disease control rates of $69 \%$ and $92 \%$ for TACE and TACE-B, respectively, were comparable to the $78 \%$ reported by another study treating CRC-LM with TACE and leucovorin, fluorouracil and oxaliplatin (FOLFOX) (22).

The results of this study showed that TACE-B was well tolerated and associated with mainly grade 1-2 adverse events that were resolved in a few days, in agreement with previous studies $(9,10)$.

The main limitations of this report were the small number of patients enrolled and the number of centers involved. Further randomized multicentric studies with a larger number of patients are necessary to confirm these data on the association of TACE with angiogenesis inhibitors in for the treatment of refractory CRC-LM.

In conclusion, the results of our study suggest that the combination of TACE with intravenous bevacizumab is effective, feasible, and well-tolerated by patients with CRCLM and may potentially have more benefits concerning tumor response and survival than TACE alone.

\section{Conflicts of Interest}

All Authors declare they have no conflicts of interest in regard to this study.

\section{Authors' Contributions}

Giammaria Fiorentini performed the bevacizumab treatment and supervised the study. Donatella Sarti collected and analyzed the data, including table and graph production, wrote the article. Stefano Guadagni supervised the study and reviewed the article. Michele 
Nardella, Riccardo Inchingolo, Massimiliano Nestola, and Alberto Rebonato performed the TACE treatments.

\section{References}

1 Lencioni R, Aliberti C, de Baere T, Garcia-Monaco R, Narayanan G, O'Grady E, Rilling WS, Walker D and Martin RC: Transarterial treatment of colorectal cancer liver metastases with irinotecan-loaded drug-eluting beads: Technical recommendations. J Vasc Interv Radiol 25(3): 365-369, 2014. PMID: 24581461. DOI: $10.1016 /$ j.jvir.2013.11.027

2 Jones RP, Jackson R, Dunne DF, Malik HZ, Fenwick SW, Poston GJ and Ghaneh P: Systematic review and meta-analysis of followup after hepatectomy for colorectal liver metastases. Br J Surg 99(4): 477-486, 2012. PMID: 22261895. DOI: 10.1002/bjs.8667

3 Hwang M, Jayakrishnan TT, Green DE, George B, Thomas JP, Groeschl RT, Erickson B, Pappas SG, Gamblin TC and Turaga KK: Systematic review of outcomes of patients undergoing resection for colorectal liver metastases in the setting of extra hepatic disease. Eur J Cancer 50(10): 1747-1757, 2014. PMID: 24767470. DOI: 10.1016/j.ejca.2014.03.277

4 Fiorentini G, Aliberti C, Tilli M, Mulazzani L, Graziano F, Giordani P, Mambrini A, Montagnani F, Alessandroni P, Catalano V and Coschiera P: Intra-arterial infusion of irinotecan-loaded drug-eluting beads (DEBIRI) versus intravenous therapy (FOLFIRI) for hepatic metastases from colorectal cancer: Final results of a phase III study. Anticancer Res 32(4): 1387-1395, 2012. PMID: 22493375.

5 Lewis AL and Holden RR: DC Bead embolic drug-eluting bead: Clinical application in the locoregional treatment of tumours. Expert Opin Drug Deliv 8(2): 153-169, 2011. PMID: 21222553. DOI: $10.1517 / 17425247.2011 .545388$

6 Aliberti C, Carandina R, Sarti D, Mulazzani L, Catalano V, Felicioli A, Coschiera P and Fiorentini G: Hepatic arterial infusion of polyethylene glycol drug-eluting beads for primary and metastatic liver cancer therapy. Anticancer Res 36(7): 35153521, 2016. PMID: 27354617.

7 Gadaleta CD and Ranier G: Trans-arterial chemoembolization as a therapy for liver tumours: new clinical developments and suggestions for combination with angiogenesis inhibitors. Crit Rev Oncol Hematol 80(1): 40-53, 2011. PMID: 21067940. DOI: 10.1016/j.critrevonc.2010.10.005

8 Ranieri G, Patruno R, Ruggieri E, Montemurro S, Valerio P and Ribatti D: Vascular endothelial growth factor (VEGF) as a target of bevacizumab in cancer: From the biology to the clinic. Curr Med Chem 13(16): 1845-1857, 2006. PMID: 16842197.

9 Ammendola M, Sacco R, Sammarco G, Donato G, Montemurro S, Ruggieri E, Patruno R, Marech I, Cariello M, Vacca A, Gadaleta CD and Ranieri G: Correlation between serum tryptase, mast cells positive to tryptase and microvascular density in colorectal cancer patients: Possible biological-clinical significance. PLoS One 9(6): e99512, 2014. PMID: 24915568. DOI: 10.1371/journal.pone.0099512

10 Marech I, Ammendola M, Gadaleta C, Zizzo N, Oakley C, Gadaleta CD and Ranieri G: Possible biological and translational significance of mast cell density in colorectal cancer. World J Gastroenterol 20(27): 8910-8920, 2014. PMID: 25083063. DOI: 10.3748/wjg.v20.i27.8910

11 Ranieri G, Coviello M, Chiriatti A, Stea B, Montemurro S, Quaranta M, Dittadi R and Paradiso A: Vascular endothelial growth factor assessment in different blood fractions of gastrointestinal cancer patients and healthy controls. Oncol Rep 11(2): 435-439, 2004. PMID: 14719080.

12 Ranieri G, Passantino L, Patruno R, Passantino G, Jirillo F, Catino A, Mattioli V, Gadaleta C and Ribatti D: The dog mast cell tumour as a model to study the relationship between angiogenesis, mast cell density and tumour malignancy. Oncol Rep 10(5): 1189-1193, 2003. PMID: 12883679.

13 Fiorentini G, Del Conte A, De Simone M, Guadagni S, Mambrini A, D'Alessandro M, Aliberti C, Rossi G and Cantore M: Complete response of colorectal liver metastases after intra-arterial chemotherapy. Tumori 94: 489-492, 2008. PMID: 18822683.

14 Buijs M, Reyes DK, Pawlik TM, Blackford AL, Salem R, Messersmith WA, Weekes CD, Mulcahy M, Kamel IR and Geschwind JF: Phase 2 trial of concurrent bevacizumab and transhepatic arterial chemoembolization in patients with unresectable hepatocellular carcinoma. Cancer 119(5): 10421049, 2013. PMID: 23132335. DOI: $10.1002 /$ cncr.27859

15 Preference Collaborative Review Group: Patients' preferences within randomised trials: Systematic review and patient level meta-analysis. BMJ 337: a1864, 2008. DOI: 10.1136/bmj.a1864

16 Chung WS, Park MS, Shin SJ, Baek SE, Kim YE, Choi JY and Kim MJ: Response evaluation in patients with colorectal liver metastases: RECIST version 1.1 versus modified CT criteria. Am J Roentgenol 199(4): 809-815, 2012. PMID: 22997372.

17 Minet E, Michel G, Remacle J and Michiels C: Role of HIF-1 as a transcription factor involved in embryonic development, cancer progression and apoptosis (review). Int J Mol Med 5: 253-259, 2000. PMID: 10677565. DOI: 10.3892/ijmm.5.3.253

18 Fiorentini G, Aliberti C, Mulazzani L, Coschiera P, Catalano V, Rossi D, Giordani P and Ricci S: Chemoembolization in colorectal liver metastases: The rebirth. Anticancer Res 34(2): 575-584, 2014. PMID: 24510986.

19 Fang P, Hu JH, Cheng ZG, Liu ZF, Wang JL and Jiao SC: Efficacy and safety of bevacizumab for the treatment of advanced hepatocellular carcinoma: A systematic review of phase II trials. PLoS One 7(12): e49717, 2012. DOI: 10.1371/journal.pone.0049717

20 Pinter M, Ulbrich G, Sieghart W, Kölblinger C, Reiberger T, Li S, Ferlitsch A, Müller C, Lammer J and Peck-Radosavljevic M: Hepatocellular carcinoma: A phase II randomized controlled double-blind trial of transarterial chemoembolization in combination with biweekly intravenous administration of bevacizumab or a placebo. Radiology 277(3): 903-912, 2015. PMID: 26131911. DOI: 10.1148/radiol.2015142140

21 Richardson AJ, Laurence JM and Lam VW: Transarterial chemoembolization with irinotecan beads in the treatment of colorectal liver metastases: systematic review. J Vasc Interv Radiol 24(8): 1209-1217, 2013. PMID: 24581461. DOI: 10.1016/j.jvir.2013.11.027

22 Jones RP, Stättner S, Dunne DF, O'Grady E, Smethurst A, Terlizzo M, Malik HZ, Fenwick SW and Poston GJ: Radiological assessment of response to neoadjuvant transcatheter hepatic therapy with irinotecan-eluting beads (DEBIRI ${ }^{\circledR}$ ) for colorectal liver metastases does not predict tumour destruction or long-term outcome. Eur J Surg Oncol 39(10): 1122-1128, 2013. PMID: 23928482. DOI: 10.1016/j.ejso.2013.07.087

Received December 3, 2019

Revised December 19, 2019 Accepted December 30, 2019 